Article

\title{
Novel 4-Methylumbelliferone Amide Derivatives: Synthesis, Characterization and Pesticidal Activities
}

\author{
Yan Wei ${ }^{\dagger}$, Kai-Long Miao ${ }^{\dagger}$ and Shuang-Hong Hao * \\ Research Center of Agro-Bionic Engineering \& Technology of Shandong Province, \\ College of Chemistry \& Pharm., Qingdao Agricultural University, Qingdao 266109, China; \\ haoyihe@sohu.com (Y.W.); m18663915715@163.com (K.-L.M.) \\ * Correspondence: doublered74@sina.com; Tel.: +86-532-8803-0522 \\ + These authors contributed equally to this article.
}

Received: 17 December 2017; Accepted: 6 January 2018; Published: 8 January 2018

\begin{abstract}
A series of novel 4-methylumbelliferone amide derivatives were designed, synthesized and characterized by ${ }^{1} \mathrm{H}$ NMR, ${ }^{13} \mathrm{C}$ NMR and HR-ESI-MS. The structures of compounds $4 \mathbf{b d}$ and 4be (compounds named by authors) were further confirmed by X-ray single crystal diffraction. The acaricidal, herbicidal and antifungal activities of the synthesized compounds were assayed for their potential use as pesticide. The results indicated that compounds $4 \mathbf{b i}, 4 \mathbf{a c}$ and $4 \mathbf{b d}$ were strong acaricidals against Tetranychus cinnabarinus, with $72 \mathrm{~h}$ corrected mortalities of greater than $80 \%$ at $1000 \mathrm{mg} / \mathrm{L}$. Meanwhile, compounds $4 \mathbf{b h}$ and $\mathbf{4 b f}$ exhibit the strongest inhibition against the taproot development of Digitaria sanguinalis and Chenopodium glaucum, and were even more potent than the commercial herbicide Acetochlor against $D$. sanguinalis. In addition, compounds $4 \mathbf{b k}, 4 \mathbf{b h}$ and $4 \mathbf{b p}$ showed the highest antifungal activity against the mycelium growth of Valsa mali, which makes them more effective than commercial fungicide Carbendazim.
\end{abstract}

Keywords: 4-methylumbelliferone; synthesis; acaricidal activity; herbicidal activity; antifungal activity

\section{Introduction}

The coumarin scaffold is an important structure type which exists in a wide class of natural and synthetic compounds [1]. Derivatives of coumarin have broad applications in the fields of perfume, food additives, cosmetics, optical brighteners [2] and dye lasers [3]. They also exhibit many bioactivities of the following categories: anticoagulant [4], vasodilator [5], sedation and hypnosis [6], analgesic and hypothermy [7], estrogenic [8], antioxidant [9], dermal photosensitization [10], antibacterial [11], antifungal [12], moluscicidal and anthelmintic [13]. Among the various coumarins, 4-methylumbelliferone (7-hydroxy-4-methylcoumarin, hymecromone) and its derivatives have been used as fluorescent probes to detect $\mathrm{Hg}^{2+}$ in neat aqueous solutions [14], and hypochlorite in tap water and cancer cells [15], as well as to assay lipases and esterases [16]. 4-Methylumbelliferone derivatives also possess diverse biological properties, such as antipsychotic [17], antidepressant [18], anaphylaxis [19], hypoglycemic [20], antioxidant [21], anti-inflammatory [22], antitumor [23,24], antibacterial [25] and antifungal [26] properties. Even though some coumarins are toxic, 4-methylumbelliferone is a safe compound used as active ingredient in several approved drugs $[27,28]$.

4-Methylumbelliferone and its derivatives exhibited interesting pesticidal activities as well. These compounds displayed strong inhibition to weeds Setaria viridis and Amaranthus retroflexus. The C7 hydroxyl group was considered as a potentially active site and a methyl substitution at the C4 position contributed significantly to the activity [29]. 4-Methylumbelliferone derivatives also showed strong growth inhibition against phytopathogenic fungi Alternaria alternata, Alternaria solani, Botrytis cinerea and Fusarium oxysporum, and a C4 methyl in the compounds contributed to the fungicidal activity [30]. Moreover, brominated 4-methylumbelliferone showed remarkable larvicidal and ovicidal 
activities against vectors Aedes aegypti and Culex quinquefasciatus [31]. 4-Methylumbelliferone esters of the chrysanthemic acid type could be metabolized by glutathione S-transferase from the mosquito Culex pipiens pipiens [32]. The Schiff base and its metal complexes of 4-methylumbelliferone derivatives showed anthelmintic to Pheretima posthuma [33].

The amide group is a common functional group in natural compounds. Many commercial pesticidal compounds have acylamino group in the molecule, for example carbamates and benzoylphenyl urea insecticides, anilide fungicides, ureas, amides and carbamates herbicides [34]. As a continuous study on the development of novel pesticides based on the coumarin scaffold [35,36], by introducing amides to 4-methylumbelliferone, a series of novel 4-methylumbelliferone amide derivatives were designed and synthesized through the principle of bioactive substructure combination. The acaricidal, herbicidal and antifungal activities of these new compounds were tested.

\section{Results and Discussion}

\subsection{Chemistry}

The synthetic route of the target compounds is illustrated in Scheme 1. Resorcinol and ethylacetoacetate occurred following a Pechmann reaction with catalyst of Con. $\mathrm{H}_{2} \mathrm{SO}_{4}$ to produce compound 1 [22]. Nitration of 4-methylumbelliferone can be achieved with $\mathrm{HNO}_{3} / \mathrm{H}_{2} \mathrm{SO}_{4}$ [37], $\mathrm{HNO}_{3} / \mathrm{HOAc}$ [37], $\mathrm{Cr}\left(\mathrm{NO}_{3}\right)_{3} / \mathrm{Ac}_{2} \mathrm{O}$ [38], $\left(\mathrm{NH}_{2}\right)_{2} \mathrm{CNH} \cdot \mathrm{HNO}_{3} / \mathrm{H}_{2} \mathrm{SO}_{4}$ [39], $\mathrm{Ce}\left(\mathrm{NH}_{4}\right)_{2}\left(\mathrm{NO}_{3}\right)_{6} /$ $\mathrm{H}_{2} \mathrm{O}_{2}$ [40] and $\mathrm{NO}_{2} \mathrm{BF}_{4}$ [41]. Each method was reported to have some regioselectivity, but it is obvious that $\mathrm{HNO}_{3} / \mathrm{HOAc}$ was more safe and accessible. Thus, in the present study, compound 1 was nitrated by Conc. $\mathrm{HNO}_{3}$ in acetic acid to give the mixture of compounds $\mathbf{2 a}$ and $\mathbf{2 b}$ [37]. Nitro 4-methylumbelliferone can be reduced by $\mathrm{SnCl}_{2} / \mathrm{HCl}$ [22], $\mathrm{Na}_{2} \mathrm{~S}_{2} \mathrm{O}_{4} / \mathrm{NH}_{3} \cdot \mathrm{H}_{2} \mathrm{O}$ [42] and D-glucose $/ \mathrm{KOH}[43]$ to give an amino product. Considering the temperature requirement, time requirements and ease of the operation, $\mathrm{Na}_{2} \mathrm{~S}_{2} \mathrm{O}_{4} / \mathrm{NH}_{3} \cdot \mathrm{H}_{2} \mathrm{O}$ was used as reductant. Thus the mixtures $\mathbf{2} \mathbf{a}$ and $\mathbf{2} \mathbf{b}$ were reduced in whole with $\mathrm{Na}_{2} \mathrm{~S}_{2} \mathrm{O}_{4}$ in aqueous $\mathrm{NH}_{3}$ to obtain the mixture of compounds $\mathbf{3 a}$ and $\mathbf{3 b}$ [41]. The target compounds $\mathbf{4 a a}-\mathbf{4} \mathbf{a h}$ and $\mathbf{4} \mathbf{b a}-\mathbf{4} \mathbf{b q}$ were furnished by the acylazation of compounds $\mathbf{3} \mathbf{a}$ and $\mathbf{3} \mathbf{b}$, respectively, with a series of acyl chlorides catalyzed by triethylamine [44]. The structures of all target compounds were well characterized by ${ }^{1} \mathrm{H} N M R,{ }^{13} \mathrm{C}$ NMR and HR-ESI-MS. Additionally, to confirm the three-dimensional structural information of the target compounds, the single-crystal structures of $\mathbf{4 b d}$ and 4 be were determined by $\mathrm{X}$-ray crystallography as illustrated in Figure 1. Crystallographic data (excluding structure factors) for the structures of $4 \mathbf{b d}$ and $4 \mathbf{b e}$ have been deposited with the Cambridge Crystallographic Data Centre as supplementary publication CCDC 1584028 and 1584029 (12 Union Road, Cambridge CB2 1EZ, UK; Fax: +44 1223 336033; E-mail: deposit@ccdc.cam.ac.uk). The C6 substituted target compounds were more polar than the C8 substituted compounds with the same group. Many fewer pure C6 substituted target compounds were created by column chromatography (CC). Moreover the $\mathrm{C} 6$ substituted target compounds 4aa-4ah were dissolved by DMSO- $d_{6}$ to determine the NMR, while the C 8 substituted compounds $\mathbf{4} \mathbf{b} \mathbf{a}-\mathbf{4} \mathbf{b q}$ were dissolved by $\mathrm{CDCl}_{3}$ for $\mathrm{NMR}$. 

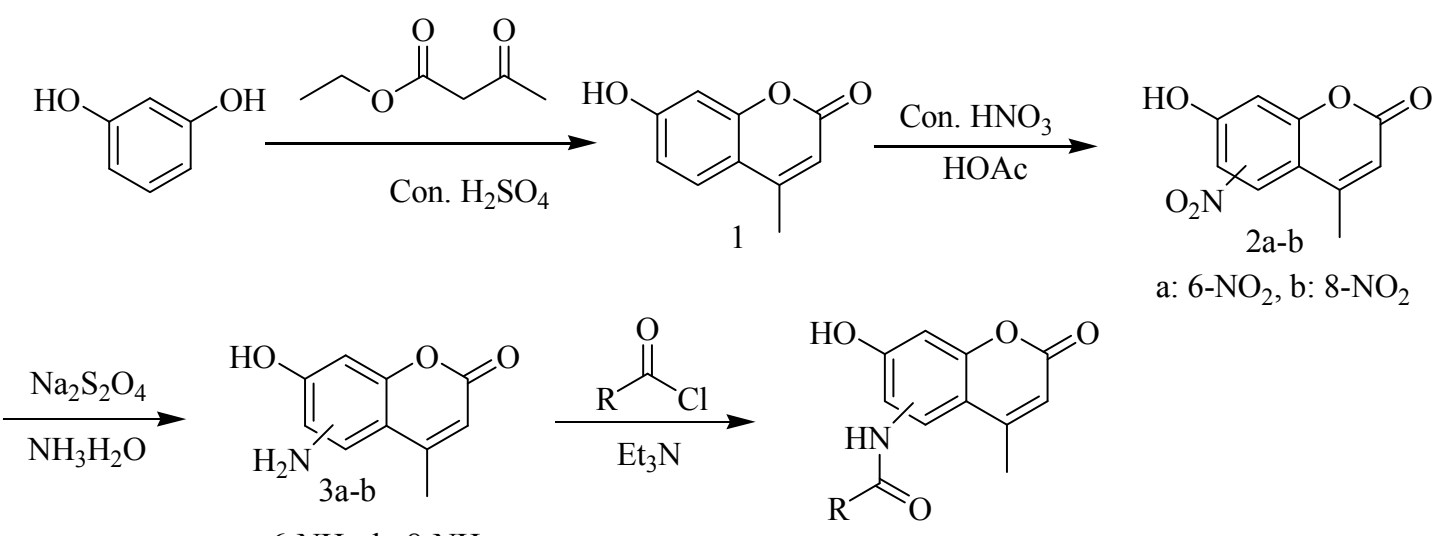

a: 6-NH 2, b: $8-\mathrm{NH}_{2}$

4aa-ah: 6-HNCOR 4ba-bq: 8-HNCOR

Compd.

Scheme 1. Synthetic route of target compounds.

\subsection{Acaricidal Activities}

The acaricidal activities of the 25 synthesized target compounds against the phytophagous mite T. cinnabarinus were evaluated. The $72 \mathrm{~h}$ corrected mortalities of the mitetreated bythe compounds are listed in Table 1. The results indicate that most of the title compounds exhibited moderate to high acaricidal potency. Among them, the most attractive compounds were $4 \mathbf{b i}$, 4 ac and $4 \mathbf{b d}$ with $72 \mathrm{~h}$ corrected mortalities of greater than $80 \%$ at $1000 \mathrm{mg} / \mathrm{L}$. Especially 4 bi showed equal toxicity with the commercial acaricide Bifenazate. In the 6-substituted derivatives, the methacryloyl substituted compound 4ac was the most active, followed by the aroyl substituted compounds 4ae-4ah. The alkanoyl substituted compounds $4 \mathbf{a a}, \mathbf{4} \mathbf{a b}$ and $4 \mathbf{a d}$ were inferior. Meanwhile, in the 
8-substituted derivatives, the most active was the hydrocinnamoyl substituted compound $4 \mathbf{b i}$, though its 6-position isomer $4 \mathbf{a d}$ and cinnyl substituted compound $\mathbf{4 b j}$ were much less potent. In the chain alkanoyl substituted compounds $\mathbf{4 b a}-\mathbf{4 b c}$, long chains seem favorable for the activity, however the 4-chloro-butanoyl substituted compound $4 \mathbf{b d}$ was an exception. The smaller cyclopropyl formyl substituted compound was more active than the larger cyclohexyl formyl substituted one. The electron withdrawing groups on the aromatic ring of the amide group were favorable for the activity, but the 4-methyl benzoyl substituted compound $4 \mathbf{b k}$ was more active.
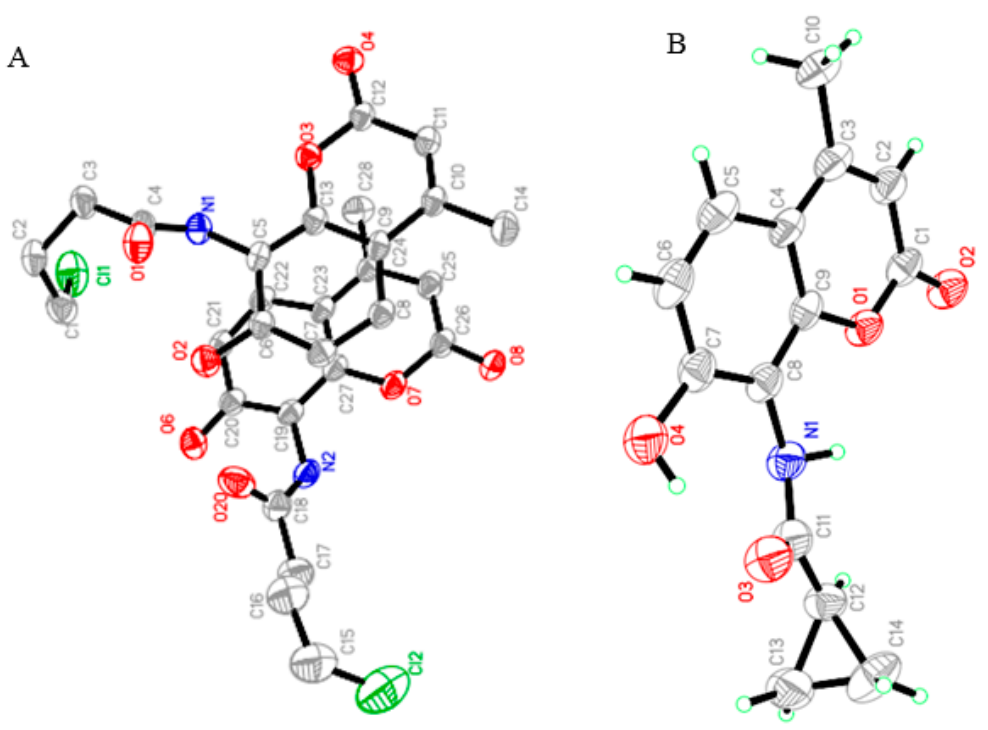

Figure 1. X-ray crystal structure of compounds $4 \mathrm{bd}(\mathrm{A})$ and $4 \mathrm{be}(\mathrm{B})$.

Table 1. Acaricidal activities of title compounds against T. cinnabarinus ( $\%, 72 \mathrm{~h}$ corrected mortality).

\begin{tabular}{cccccc}
\hline Compd. & $\mathbf{2 0 0} \mathbf{~} \mathbf{g} / \mathbf{L}$ & $\mathbf{1 0 0 0} \mathbf{~} \mathbf{~ g} / \mathbf{L}$ & Compd. & $\mathbf{2 0 0} \mathbf{~} \mathbf{g} / \mathbf{L}$ & $\mathbf{1 0 0 0} \mathbf{~} \mathbf{g} / \mathbf{L}$ \\
\hline $\mathbf{4 a a}$ & $4.2 \pm 5.2$ & $12.9 \pm 5.0$ & $\mathbf{4 b f}$ & $18.4 \pm 3.0$ & $29.2 \pm 6.3$ \\
$\mathbf{4 a b}$ & $4.5 \pm 3.2$ & $27.1 \pm 6.5$ & $\mathbf{4 b g}$ & $40.3 \pm 3.7$ & $71.7 \pm 5.4$ \\
$\mathbf{4 a c}$ & $51.9 \pm 9.9$ & $88.3 \pm 4.9$ & $\mathbf{4 b h}$ & $54.0 \pm 13.1$ & $65.1 \pm 6.6$ \\
$\mathbf{4 a d}$ & $9.1 \pm 5.0$ & $19.4 \pm 2.7$ & $\mathbf{4 b i}$ & $87.8 \pm 8.1$ & $98.6 \pm 6.1$ \\
$\mathbf{4 a e}$ & $23.0 \pm 4.9$ & $42.1 \pm 10.9$ & $\mathbf{4 b j}$ & $26.6 \pm 5.8$ & $38.0 \pm 8.0$ \\
$\mathbf{4 a f}$ & $33.9 \pm 2.9$ & $56.9 \pm 4.7$ & $\mathbf{4 b k}$ & $57.4 \pm 10.9$ & $79.8 \pm 8.3$ \\
$\mathbf{4 a g}$ & $32.8 \pm 5.6$ & $52.6 \pm 7.3$ & $\mathbf{4 b 1}$ & $23.0 \pm 6.3$ & $31.7 \pm 9.7$ \\
$\mathbf{4 a h}$ & $30.0 \pm 3.5$ & $53.1 \pm 7.5$ & $\mathbf{4 b m}$ & $7.1 \pm 8.6$ & $55.0 \pm 10.7$ \\
$\mathbf{4 b a}$ & $20.4 \pm 9.6$ & $30.0 \pm 3.4$ & $\mathbf{4 b n}$ & $47.4 \pm 10.3$ & $58.8 \pm 7.4$ \\
$\mathbf{4 b b}$ & $11.8 \pm 7.1$ & $29.7 \pm 10.0$ & $\mathbf{4 b o}$ & $53.8 \pm 8.0$ & $63.4 \pm 4.6$ \\
$\mathbf{4 b c}$ & $61.5 \pm 2.4$ & $69.2 \pm 2.4$ & $\mathbf{4 b p}$ & $48.7 \pm 9.5$ & $62.6 \pm 3.0$ \\
$\mathbf{4 b d}$ & $75.1 \pm 7.6$ & $83.5 \pm 5.6$ & $\mathbf{4 b q}$ & $41.9 \pm 3.0$ & $72.6 \pm 4.2$ \\
$\mathbf{4 b e}$ & $65.1 \pm 3.2$ & $73.8 \pm 2.3$ & Bifenazate & $93.5 \pm 5.7$ & $100.0 \pm 0.0$ \\
\hline
\end{tabular}

\subsection{Herbicidal Activities}

The herbicidal activities of the target compounds against the taproot and caulis development of dicotyledonous weed C. glaucum and monocotyledonous weed D. sanguinalis were screened. The inhibitory rates of the compounds an effectiveness greater than $30 \%$ at $100 \mathrm{mg} / \mathrm{L}$ to at least one organ of the weeds are displayed in Table 2. The data indicate that more compounds show stronger inhibition against D. sanguinalis than against C. glaucum. More specifically, compounds $4 \mathbf{b h}$ and $4 \mathbf{b f}$ exhibit the strongest inhibition against the taproot development of $D$. sanguinalis, which makes them even more potent than the commercial herbicide Acetochlor. In fact $\mathbf{4 b h}$ and $\mathbf{4 b f}$ were the most potent against the taproot development of C. glaucum. Regarding effectiveness against $D$. sanguinalis, the chain 
alkanoylsubstituted compounds $\mathbf{4 b a}-\mathbf{4 b d}$ (long chain compounds) seem unfavorable for the activity. Meanwhile the larger cyclohexyl formyl substituted compound was more active than the smaller cyclopropyl formyl substituted one.

Table 2. Herbicidal activities of target compounds (\%, $100 \mathrm{mg} / \mathrm{L})$.

\begin{tabular}{|c|c|c|c|c|c|c|c|c|}
\hline Compd. & \multicolumn{2}{|c|}{ C. glaucum } & Compd. & \multicolumn{2}{|c|}{ D. sanguinalis } & Compd. & \multicolumn{2}{|c|}{ D. sanguinalis } \\
\hline 4 af & $31.2 \pm 9.7$ & - & $4 \mathbf{a a}$ & $41.6 \pm 2.6$ & $67.4 \pm 4.4$ & $4 b j$ & $10.6 \pm 5.7$ & $61.5 \pm 12.4$ \\
\hline 4ah & - & $40.0 \pm 5.3$ & $4 a b$ & $17.4 \pm 7.4$ & $33.6 \pm 9.1$ & $4 \mathrm{bk}$ & $41.9 \pm 8.5$ & $54.8 \pm 8.7$ \\
\hline $4 \mathrm{bb}$ & $37.1 \pm 9.6$ & $16.8 \pm 6.5$ & 4ad & $25.5 \pm 4.4$ & $53.1 \pm 8.9$ & $4 \mathrm{bl}$ & $27.8 \pm 5.2$ & $48.5 \pm 7.9$ \\
\hline $4 b c$ & $40.1 \pm 8.6$ & $15.3 \pm 6.5$ & 4ag & $30.6 \pm 3.6$ & - & $4 \mathrm{bm}$ & $44.8 \pm 7.7$ & $12.2 \pm 13.0$ \\
\hline $4 \mathrm{bh}$ & $76.3 \pm 3.3$ & $39.3 \pm 1.9$ & $4 b c$ & - & $45.4 \pm 11.7$ & $4 \mathrm{bq}$ & $54.8 \pm 4.3$ & $60.9 \pm 10.9$ \\
\hline 4bk & $41.8 \pm 6.0$ & - & $4 \mathrm{bd}$ & $3.4 \pm 12.1$ & $62.6 \pm 10.4$ & Acetochlor & $84.2 \pm 7.0$ & $78.1 \pm 8.4$ \\
\hline $4 \mathrm{bm}$ & $51.1 \pm 7.9$ & - & $4 \mathrm{be}$ & $18.9 \pm 6.8$ & $40.5 \pm 5.6$ & & & \\
\hline $4 b n$ & $31.1 \pm 2.2$ & $10.9 \pm 8.1$ & $4 b f$ & $86.4 \pm 10.6$ & $71.8 \pm 8.1$ & & & \\
\hline 4 bo & $30.1 \pm 2.6$ & $63.0 \pm 5.8$ & $4 \mathrm{bg}$ & $44.4 \pm 11.7$ & - & & & \\
\hline
\end{tabular}

\subsection{Antifungal Activities}

The in vitro antifungal activities of the target compounds against the mycelium growth of the phytopathogens Colletotrichum glecosporioides, B. cinerea, F. oxysporum and V. mali were assayed. The inhibitory rates of the compounds with effectiveness greater than $30 \%$ at $100 \mathrm{mg} / \mathrm{L}$ are listed in Table 3. The data indicate that more compounds showed stronger inhibition against $V$. mali than against the other 3 plant disease fungi. More specifically, compounds $4 \mathbf{b k}, \mathbf{4 b h}$ and $4 \mathbf{b p}$ exhibited the strongest inhibition against the mycelium growth of $V$. mali, which makes them even more potent than the commercial fungicide Carbendazim. Furthermore, the effectiveness of compounds $4 \mathbf{b e}, 4 \mathbf{b i}$ and $\mathbf{4 b d}$ against $B$. cinerea were superior than or comparable with that of the fungicide Carbendazim, though they are all only moderately active against the fungi. The inhibition of compound $4 \mathbf{b q}$ against F. oxysporium was comparable with that of fungicide Carbendazim.

Table 3. Antifungal activities of target compounds (\%, $100 \mathrm{mg} / \mathrm{L})$.

\begin{tabular}{cccccccccc}
\hline Compd. & F1 & Compd. & F2 & Compd. & F3 & Compd. & F4 & Compd. & F4 \\
\hline $\mathbf{4 b f}$ & $45.8 \pm 8.6$ & $\mathbf{4 a b}$ & $48.2 \pm 7.4$ & $\mathbf{4 a c}$ & $31.8 \pm 7.9$ & $\mathbf{4 a b}$ & $32.6 \pm 1.1$ & $\mathbf{4 b j}$ & $39.8 \pm 2.1$ \\
$\mathbf{4 b h}$ & $32.1 \pm 5.5$ & $\mathbf{4 a g}$ & $45.9 \pm 9.0$ & $\mathbf{4 b h}$ & $38.4 \pm 3.3$ & $\mathbf{4 a e}$ & $32.6 \pm 8.9$ & $\mathbf{4 b k}$ & $88.7 \pm 1.9$ \\
$\mathbf{4 b k}$ & $56.6 \pm 5.0$ & $\mathbf{4 b d}$ & $54.6 \pm 7.8$ & $\mathbf{4 b j}$ & $36.1 \pm 4.2$ & $\mathbf{4 b a}$ & $47.3 \pm 1.6$ & $\mathbf{4 b \mathbf { b }}$ & $48.1 \pm 2.2$ \\
$\mathbf{4 b \mathbf { b }}$ & $39.3 \pm 6.1$ & $\mathbf{4 b e}$ & $69.4 \pm 6.2$ & $\mathbf{4 b k}$ & $46.6 \pm 1.9$ & $\mathbf{4 b d}$ & $36.5 \pm 1.6$ & $\mathbf{4 b m}$ & $51.0 \pm 2.5$ \\
$\mathbf{4 b q}$ & $60.8 \pm 3.6$ & $\mathbf{4 b f}$ & $32.9 \pm 9.8$ & $\mathbf{4 b n}$ & $48.9 \pm 4.1$ & $\mathbf{4 b f}$ & $46.5 \pm 4.0$ & $\mathbf{4 b n}$ & $72.7 \pm 4.1$ \\
Carb. & $88.9 \pm 4.7$ & $\mathbf{4 b i}$ & $57.8 \pm 3.8$ & $\mathbf{4 b p}$ & $47.9 \pm 4.2$ & $\mathbf{4 b g}$ & $66.1 \pm 5.2$ & $\mathbf{4 b o}$ & $34.8 \pm 8.6$ \\
& & $\mathbf{4 b \mathbf { b }}$ & $34.5 \pm 9.9$ & $\mathbf{4 b q}$ & $71.2 \pm 4.8$ & $\mathbf{4 b h}$ & $84.9 \pm 1.6$ & $\mathbf{4 b \mathbf { b }}$ & $81.8 \pm 4.2$ \\
& & $\mathbf{4 b n}$ & $37.7 \pm 6.8$ & Carb. & $70.9 \pm 8.4$ & $\mathbf{4 b i}$ & $58.4 \pm 2.7$ & $\mathbf{4 b q}$ & $61.1 \pm 4.8$ \\
& & Carb. & $54.1 \pm 7.1$ & & & & & Carb. & $76.1 \pm 3.7$ \\
\hline
\end{tabular}

F1: C. glecosporioides; F2: B. cinerea; F3: F. oxysporum; F4: V. mali; Carb.: Carbendazim.

\section{Experimental Section}

\subsection{Chemistry}

All chemicals were obtained from commercial sources and used without further purification. Analytical thin-layer chromatography (TLC) was performed with silica gel plates using silica gel 60 $\mathrm{GF}_{254}$ (Qingdao Haiyang Chemical Co., Ltd., Qingdao, China). Melting points were determined on a WRS-1B digital melting-point apparatus (Shanghai Precision Optical Instrument Co., Ltd., Shanghai, China) without further calibration. Nuclear magnetic resonance spectra (NMR) were 
recorded on a Bruker Avance III HD $500 \mathrm{MHz}$ instrument (Bruker, Faellanden, Switzerland) in $\mathrm{CDCl}_{3}$ or DMSO- $d_{6}\left({ }^{1} \mathrm{H}\right.$ at $500 \mathrm{MHz}$ and ${ }^{13} \mathrm{C}$ at $\left.126 \mathrm{MHz}\right)$ using tetramethylsilane (TMS) as the internal standard. High-resolution mass spectra (HRMS) were carried out with an IonSpec $4.7 \mathrm{~T}$ FTMS instrument. Single-crystal structure was determined by a Bruker AXS D8 QUEST X-ray single crystal diffractometer.

\subsubsection{Synthesis of $\mathbf{1}$}

A solution of resorcinol $(5.5 \mathrm{~g}, 50 \mathrm{mmol})$ and ethyl acetoacetate $(6.5 \mathrm{~g}, 50 \mathrm{mmol})$ in ethanol $(10 \mathrm{~mL})$ was added dropwise to $\mathrm{H}_{2} \mathrm{SO}_{4}(5 \mathrm{~mL})$ with stirring at $0-5{ }^{\circ} \mathrm{C}$. After the complete addition, the reaction mixture was stirred for $4 \mathrm{~h}$ at room temperature. Then the reaction mixture was poured onto ice-water $(100 \mathrm{~mL})$ with vigorous stirring for $1 \mathrm{~h}$. The white precipitate which formed was collected, and washed with cold water to neutral. The product was dried and crystallized, and separated from ethanol. Yield $88 \%$, m.p. $185-186^{\circ} \mathrm{C}$ (as reported) [22].

\subsubsection{Synthesis of $\mathbf{2 a}$ and $\mathbf{2 b}$}

To a solution of $\mathbf{1}(8.8 \mathrm{~g}, 50 \mathrm{mmol})$ in acetic acid $(20 \mathrm{~mL})$ kept at a temperature below $10{ }^{\circ} \mathrm{C}$, a mixture of $65 \%$ nitric acid $(5.8 \mathrm{~g}, 60 \mathrm{mmol})$ and acetic acid $(20 \mathrm{~mL})$ was added dropwise with stirring. After the complete addition, the reaction mixture was stirred for another $6 \mathrm{~h}$ at room temperature and then poured onto ice-water. The orange precipitate obtained was filtered off, washed with water to neutral, and air-dried. The solid product was washed with hot acetonitrile to give the yellow mixtures $\mathbf{2 a}$ and $\mathbf{2 b}$ (3:1) with a yield of $70 \%$ [37].

\subsubsection{Synthesis of $\mathbf{3 a}$ and $\mathbf{3 b}$}

To a suspension of $\mathbf{2 a}$ and $\mathbf{2} \mathbf{b}$ mixtures $(11.1 \mathrm{~g}, 50 \mathrm{mmol})$ in $60 \mathrm{~mL}$ of concentrated aqueous ammonia, $70 \mathrm{~mL}$ of $15 \%$ sodium hydrosulfite was added slowly with stirring at room temperature. After the complete addition, the reaction mixture was stirred for $6 \mathrm{~h}$ until the color changed from bright orange to light green. The mixture was then boiled for $15 \mathrm{~min}$, cooled to $5{ }^{\circ} \mathrm{C}$, and the solid was filtered off to give the mixtures $\mathbf{3} \mathbf{a}$ and $\mathbf{3 b}$ (3:1) with a yield of $93 \%$ [41].

3.1.4. General Procedure for Synthesis of 4-Methylumbelliferone Amide Derivatives (4aa-4ah, 4ba-4bq)

To a suspension of $1.91 \mathrm{~g}$ of 6-amino-7-hydroxyl-4-methylcourmin $(10 \mathrm{mmol})$ and $100 \mathrm{uL}$ of triethylamine in $30 \mathrm{~mL}$ of dichloromethane (DCM), a solution of acyl chloride (10 mmol) in $20 \mathrm{~mL}$ $\mathrm{DCM}$ was added with stirring at $0-5{ }^{\circ} \mathrm{C}$. The mixture was allowed to restore to room temperature and was stirred until the end of the reaction. The resulting organic mixture was washed with $30 \mathrm{~mL}$ of water (producing $\mathrm{Na}_{2} \mathrm{SO}_{4}$ ), then dried, filtered and concentrated in vacuo sequentially. The obtained residue was purified by silica gel column chromatography (petroleum ether-ethyl acetate $=1: 1$ for $\mathbf{4 a a}-\mathbf{4 a h}$, petroleum ether-ethyl acetate $=4: 1$ for $\mathbf{4 b a}-\mathbf{4 b q}$ ) to give the target compounds [44]. The yield, appearance, m.p., the ${ }^{1} \mathrm{H}-\mathrm{NMR}$ and ${ }^{13} \mathrm{C}$ NMR spectra of Compounds $4 \mathbf{a a}-\mathbf{4} \mathbf{b q}$ as Supplementary Materials, NMR and HRMS dates of the synthesized compounds are listed below.

6-(n-Butanoylamino)-4-methylumbelliferone (4aa). Pale yellow solid; yield: 71.2\%; m.p. 251.6-254.8 ${ }^{\circ} \mathrm{C}$; ${ }^{1} \mathrm{H}$ NMR $\delta: 11.10(\mathrm{~s}, 1 \mathrm{H}), 9.27(\mathrm{~s}, 1 \mathrm{H}), 8.24(\mathrm{~s}, 1 \mathrm{H}), 6.83(\mathrm{~s}, 1 \mathrm{H}), 6.18(\mathrm{~s}, 1 \mathrm{H}), 2.40(\mathrm{t}, J=7.0 \mathrm{~Hz}, 2 \mathrm{H}), 2.35$ (s, 3H), 1.67-1.58 (m, 2H), $0.93(\mathrm{t}, J=7.5 \mathrm{~Hz}, 3 \mathrm{H}) ;{ }^{13} \mathrm{C}$ NMR $\delta: 172.29,160.77,153.87,152.55,151.05$, 124.51, 118.06, 111.81, 111.26, 102.66, 38.29, 19.09, 18.66, 14.13; HR-ESI-MS m/z: 260.0918 [M - H] ${ }^{-}$ (calculated for $\mathrm{C}_{14} \mathrm{H}_{14} \mathrm{NO}_{4}, 260.0923$ ).

6-(Cyclohexyl-formylamino)-4-methylumbelliferone (4ab). Yellow solid; yield: 56.3\%; m.p. 116.5-117.8 ${ }^{\circ} \mathrm{C}$; ${ }^{1} \mathrm{H}$ NMR $\delta: 11.08(\mathrm{~s}, 1 \mathrm{H}), 9.15(\mathrm{~s}, 1 \mathrm{H}), 8.25(\mathrm{~s}, 1 \mathrm{H}), 6.81(\mathrm{~s}, 1 \mathrm{H}), 6.16(\mathrm{~s}, 1 \mathrm{H}), 2.58-2.53(\mathrm{~m}, 1 \mathrm{H}), 2.34$ $(\mathrm{s}, 3 \mathrm{H}), 1.82-1.73(\mathrm{~m}, 3 \mathrm{H}), 1.66-1.63(\mathrm{~m}, 1 \mathrm{H}), 1.44\left(\mathrm{qd}, J_{1}=12 \mathrm{~Hz}, J_{2}=2.5 \mathrm{~Hz}, 2 \mathrm{H}\right), 1.32-1.13(\mathrm{~m}, 4 \mathrm{H})$; 
${ }^{13}$ C NMR $8:$ 175.37, 160.74, 153.85, 152.39, 150.96, 124.62, 117.73, 111.81, 111.26, 102.65, 44.68, 29.76, 25.66, 18.66; HR-ESI-MS $m / z: 300.1232[\mathrm{M}-\mathrm{H}]^{-}$(calculated for $\mathrm{C}_{17} \mathrm{H}_{18} \mathrm{NO}_{4}, 300.1236$ ).

6-(2-Methyl-acryloylamino)-4-methylumbelliferone (4ac). Pale yellow solid; yield: 53.4\%; m.p.

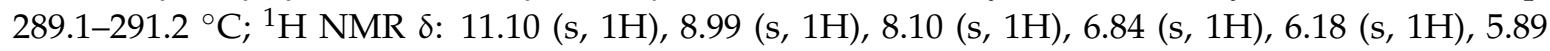
(s, 1H), $5.54(\mathrm{~s}, 1 \mathrm{H}), 2.36(\mathrm{~s}, 3 \mathrm{H}), 1.99(\mathrm{~s}, 3 \mathrm{H}) ;{ }^{13} \mathrm{C}$ NMR $\delta: 166.80,160.71,153.82,153.60,151.73$, $140.19,123.92,121.26,119.45,111.93,111.35,102.81,18.98,18.67$. HR-ESI-MS $m / z: 260.0926[\mathrm{M}+\mathrm{H}]^{+}$ (calculated for $\mathrm{C}_{14} \mathrm{H}_{14} \mathrm{NO}_{4}, 260.0923$ ).

6-Hydrocinnamoylamino-4-methylumbelliferone (4ad). White solid; yield: 59.2\%; m.p. 245.5-247.1 ${ }^{\circ} \mathrm{C}$; ${ }^{1} \mathrm{H}$ NMR $\delta: 11.10(\mathrm{~s}, 1 \mathrm{H}), 9.37(\mathrm{~s}, 1 \mathrm{H}), 8.24(\mathrm{~s}, 1 \mathrm{H}), 7.31-7.26(\mathrm{~m}, 4 \mathrm{H}), 7.21-7.17(\mathrm{~m}, 1 \mathrm{H}), 6.84(\mathrm{~s}, 1 \mathrm{H})$, $6.16(\mathrm{~s}, 1 \mathrm{H}), 2.91(\mathrm{t}, J=20.8 \mathrm{~Hz}, 2 \mathrm{H}), 2.75(\mathrm{~s}, J=18.75 \mathrm{~Hz}, 2 \mathrm{H}), 2.34(\mathrm{~s}, 3 \mathrm{H}) ;{ }^{13} \mathrm{C}$ NMR $\delta: 171.55,160.80$, 153.86, 152.73, 151.07, 141.64, 128.78, 126.41, 124.49, 117.91, 111.68, 111.16, 102.66, 37.93, 31.41, 18.66 . HR-ESI-MS m/z: $324.1235[\mathrm{M}+\mathrm{H}]^{+}$(calculated for $\mathrm{C}_{19} \mathrm{H}_{18} \mathrm{NO}_{4}, 324.1236$ ).

6-(4-Methyl-benzoylamino)-4-methylumbelliferone (4ae). White solid; yield: 44.7\%; m.p. 286.8-288.1 ${ }^{\circ} \mathrm{C}$; ${ }^{1} \mathrm{H}$ NMR $\delta: 11.09$ (s, 1H), $9.54(\mathrm{~s}, 1 \mathrm{H}), 8.07$ (s, 1H), $7.90(\mathrm{~s}, 2 \mathrm{H}), 7.34(\mathrm{~s}, 2 \mathrm{H}), 6.88(\mathrm{~s}, 1 \mathrm{H}), 6.19(\mathrm{~s}, 1 \mathrm{H}), 2.38$ (s, 6H); ${ }^{13} \mathrm{C}$ NMR $\delta: 165.72,160.74,154.44,153.86,152.05,142.30,129.54,128.07,123.94,120.81,111.99$, 111.31, 103.01, 21.51, 18.69; HR-ESI-MS $m / z: 308.0923$ [M - H] ${ }^{-}$(calculated for $\mathrm{C}_{18} \mathrm{H}_{14} \mathrm{NO}_{4}, 308.0923$ ).

6-(3-Fluoro-benzoylamino)-4-methylumbelliferone (4af). Yellow solid; yield: 49.2\%; m.p. 228.8-229.5 ${ }^{\circ} \mathrm{C}$;

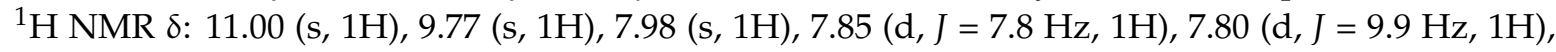
$7.60\left(\mathrm{qd}, J_{1}=6.0 \mathrm{~Hz}, J_{2}=2.0 \mathrm{~Hz}, 1 \mathrm{H}\right), 7.46\left(\mathrm{td}, J_{1}=8.5 \mathrm{~Hz}, J_{2}=2.0 \mathrm{~Hz}, 1 \mathrm{H}\right), 6.88(\mathrm{~s}, 1 \mathrm{H}), 6.20(\mathrm{~s}, 1 \mathrm{H})$, 2.38 (s, 3H); ${ }^{13} \mathrm{C}$ NMR $\delta: ~ 164.66,162.49,160.71,155.04,153.84,152.45,137.09,131.17,124.31,123.39$, 121.96, 119.12, 114.99, 112.06, 111.37, 103.12, 18.70; HR-ESI-MS m/z: $314.0828[\mathrm{M}+\mathrm{H}]^{+}$(calculated for $\left.\mathrm{C}_{17} \mathrm{H}_{13} \mathrm{FNO}_{4}, 314.0829\right)$.

6-(Furyl-2-formylamino)-4-methylumbelliferone (4ag). White solid; yield: 43.4\%; m.p. 298.5-299.7 ${ }^{\circ} \mathrm{C}$; ${ }^{1} \mathrm{H}$ NMR $\delta: 11.23(\mathrm{~s}, 1 \mathrm{H}), 9.27(\mathrm{~s}, 1 \mathrm{H}), 8.21(\mathrm{~s}, 1 \mathrm{H}), 7.95(\mathrm{~s}, 1 \mathrm{H}), 7.31(\mathrm{~s}, 1 \mathrm{H}), 6.88(\mathrm{~s}, 1 \mathrm{H}), 6.72(\mathrm{~s}, 1 \mathrm{H})$, $6.20(\mathrm{~s}, 1 \mathrm{H}), 2.37(\mathrm{~s}, 3 \mathrm{H}) ;{ }^{13} \mathrm{C}$ NMR $\delta:$ 160.68, 156.49, 153.78, 153.25, 151.78, 147.75, 146.35, 123.39, $118.94,115.48,112.90,112.05,111.46,102.81,18.63$; HR-ESI-MS $m / z: 284.0563$ [M - H] ${ }^{-}$(calculated for $\left.\mathrm{C}_{15} \mathrm{H}_{10} \mathrm{NO}_{5}, 284.0559\right)$.

6-(Thienyl-2-formylamino)-4-methylumbelliferone (4ah). White solid; yield: $53.4 \%$; m.p. $253.2-254.8{ }^{\circ} \mathrm{C}$; ${ }^{1} \mathrm{H}$ NMR $\delta: 10.99(\mathrm{~s}, 1 \mathrm{H}), 9.68(\mathrm{~s}, 1 \mathrm{H}), 8.01(\mathrm{~d}, J=3.5 \mathrm{~Hz}, 1 \mathrm{H}), 7.93(\mathrm{~s}, 1 \mathrm{H}), 7.86(\mathrm{~d}, J=4.9 \mathrm{~Hz}, 1 \mathrm{H}), 7.22(\mathrm{t}$, $J=4.5 \mathrm{~Hz}, 1 \mathrm{H}), 6.87(\mathrm{~s}, 1 \mathrm{H}), 6.19(\mathrm{~s}, 1 \mathrm{H}), 2.37(\mathrm{~s}, 3 \mathrm{H}) ;{ }^{13} \mathrm{C}$ NMR $\delta: 160.66,160.64,154.88,153.77,152.33$, 139.91, 132.27, 129.77, 128.62, 123.20, 121.86, 112.05, 111.33, 103.08, 18.63; HR-ESI-MS m/z: 300.0328 $[\mathrm{M}-\mathrm{H}]^{-}$(calculated for $\mathrm{C}_{15} \mathrm{H}_{10} \mathrm{NO}_{4} \mathrm{~S}, 300.0331$ ).

8-(n-Butanoylamino)-4-methylumbelliferone (4ba). Pale yellow solid; yield: $17.2 \%$; m.p. $141.1-143.4{ }^{\circ} \mathrm{C}$; ${ }^{1} \mathrm{H}$ NMR $\delta: 10.72(\mathrm{~s}, 1 \mathrm{H}), 8.09(\mathrm{~s}, 1 \mathrm{H}), 7.36(\mathrm{~d}, J=9.0 \mathrm{~Hz}, 1 \mathrm{H}), 6.96(\mathrm{~d}, J=9.0 \mathrm{~Hz}, 1 \mathrm{H}), 6.13(\mathrm{~s}, 1 \mathrm{H}), 2.57$ $(\mathrm{t}, J=7.5 \mathrm{~Hz}, 2 \mathrm{H}), 2.42(\mathrm{~s}, 3 \mathrm{H}), 1.86-1.79(\mathrm{~m}, 2 \mathrm{H}), 1.06(\mathrm{t}, J=7.5 \mathrm{~Hz}, 3 \mathrm{H}) ;{ }^{13} \mathrm{C}$ NMR $\delta: 173.58,158.83$, $152.67,151.43,144.56,121.20,115.38,113.00,111.56,110.03,37.86,18.23,17.95,12.56$; HR-ESI-MS $m / z$ : $260.0921[\mathrm{M}-\mathrm{H}]^{-}$(calculated for $\mathrm{C}_{14} \mathrm{H}_{14} \mathrm{NO}_{4}, 260.0923$ ).

8-(i-Butanoylamino)-4-methylumbelliferone (4bb). White solid; yield: $46.8 \%$; m.p.121.5-122.6 ${ }^{\circ} \mathrm{C} ;{ }^{1} \mathrm{H}$ NMR $\delta: 10.83(\mathrm{~s}, 1 \mathrm{H}), 8.18(\mathrm{~s}, 1 \mathrm{H}), 7.36(\mathrm{~d}, J=8.9 \mathrm{~Hz}, 1 \mathrm{H}), 6.95(\mathrm{~d}, J=8.9 \mathrm{~Hz}, 1 \mathrm{H}), 6.13(\mathrm{~s}, 1 \mathrm{H}), 2.85-2.81$ $(\mathrm{m}, 1 \mathrm{H}), 2.42(\mathrm{~s}, 3 \mathrm{H}), 1.34(\mathrm{~d}, J=6.9 \mathrm{~Hz}, 6 \mathrm{H}) ;{ }^{13} \mathrm{C}$ NMR $\delta: 178.65,159.85,153.70,152.46,145.69,122.18$, $116.37,114.00,112.56,111.02,36.27,19.73,18.96$; HR-ESI-MS $m / z: 262.1081[\mathrm{M}+\mathrm{H}]^{+}$(calculated for $\left.\mathrm{C}_{14} \mathrm{H}_{16} \mathrm{NO}_{4}, 262.1079\right)$.

8-(Lauroylamino)-4-methylumbelliferone (4bc). White solid; yield: $25.2 \%$; m.p. $149.2-151.8^{\circ} \mathrm{C} ;{ }^{1} \mathrm{H}$ NMR $\delta$ : $10.75(\mathrm{~s}, 1 \mathrm{H}), 8.11(\mathrm{~s}, 1 \mathrm{H}), 7.36(\mathrm{~d}, J=8.2 \mathrm{~Hz}, 1 \mathrm{H}), 6.96(\mathrm{~d}, J=9.4 \mathrm{~Hz}, 1 \mathrm{H}), 6.14(\mathrm{~s}, 1 \mathrm{H}), 2.58(\mathrm{t}, J=5.0 \mathrm{~Hz}$, 2H), $2.42(\mathrm{~s}, 3 \mathrm{H}), 1.78(\mathrm{~m}, 2 \mathrm{H}), 1.41-1.26(\mathrm{~m}, 16 \mathrm{H}), 0.88(\mathrm{t}, J=5.0 \mathrm{~Hz}, 3 \mathrm{H}) ;{ }^{13} \mathrm{C}$ NMR $\delta: 174.87,159.94$, 
153.76, 152.47, 145.58, 122.21, 116.44, 114.08, 112.58, 111.04, 37.09, 31.92, 29.60, 29.44, 29.34, 29.28, 29.10, 25.75, 22.70, 18.99, 14.13; HR-ESI-MS $m / z: 396.2146[\mathrm{M}+\mathrm{Na}]^{+}$(calculated for $\mathrm{C}_{22} \mathrm{H}_{31} \mathrm{NNaO}_{4}, 396.2151$ ).

8-(4-Chloro-butanoylamino)-4-methylumbelliferone (4bd). White solid; yield: 55.7\%; m.p. 155.2-156. ${ }^{\circ} \mathrm{C}$; ${ }^{1} \mathrm{H}$ NMR $\delta: 10.41(\mathrm{~s}, 1 \mathrm{H}), 8.30(\mathrm{~s}, 1 \mathrm{H}), 7.36(\mathrm{~d}, J=8.8 \mathrm{~Hz}, 1 \mathrm{H}), 6.96(\mathrm{~d}, J=8.8 \mathrm{~Hz}, 1 \mathrm{H}), 6.13(\mathrm{~s}, 1 \mathrm{H}), 3.68$ $(\mathrm{t}, J=6.1 \mathrm{~Hz}, 2 \mathrm{H}), 2.82(\mathrm{t}, J=7.1 \mathrm{~Hz}, 2 \mathrm{H}), 2.41(\mathrm{~s}, 3 \mathrm{H}), 2.28-2.22(\mathrm{~m}, 2 \mathrm{H}) ;{ }^{13} \mathrm{C}$ NMR $\delta: 173.45,159.90$, 153.71, 152.50, 145.73, 122.43, 116.41, 113.90, 112.70, 111.15, 43.92, 33.60, 27.97, 18.96; HR-ESI-MS $m / z$ : 294.0541, $296.0497[\mathrm{M}-\mathrm{H}]^{-}$(calculated for $\left.\mathrm{C}_{14} \mathrm{H}_{13} \mathrm{ClNO}_{4}, 294.0533,296.0504\right)$.

8-(Cyclopropyl-formylamino)-4-methylumbelliferone (4be). Yellow solid; yield: $31.2 \%$; m.p. $162.5-163.7^{\circ} \mathrm{C}$; ${ }^{1} \mathrm{H}$ NMR $\delta: 10.82(\mathrm{~s}, 1 \mathrm{H}), 8.40(\mathrm{~s}, 1 \mathrm{H}), 7.34(\mathrm{~d}, J=8.9 \mathrm{~Hz}, 1 \mathrm{H}), 6.94(\mathrm{~d}, J=8.8 \mathrm{~Hz}, 1 \mathrm{H}), 6.13(\mathrm{~s}, 1 \mathrm{H}), 2.41(\mathrm{~s}$, $3 \mathrm{H}), 1.90-1.86(\mathrm{~m}, 1 \mathrm{H}), 1.22-1.16(\mathrm{~m}, 2 \mathrm{H}), 1.04-1.00(\mathrm{~m}, 2 \mathrm{H}) ;{ }^{13} \mathrm{C}$ NMR $\delta: 175.23,160.08,153.79,152.50$, 145.46, 121.97, 116.38, 114.34, 112.53, 110.94, 18.95, 15.60, 9.48; HR-ESI-MS m/z: $260.0921\left[\mathrm{M}+\mathrm{H}^{+}\right.$ (calculated for $\mathrm{C}_{14} \mathrm{H}_{14} \mathrm{NO}_{4}, 260.0923$ ).

8-(Cyclohexyl-formylamino)-4-methylumbelliferone (4bf). Yellow solid; yield: $14.1 \%$; m.p. $156.3-158.4{ }^{\circ} \mathrm{C}$; ${ }^{1} \mathrm{H}$ NMR $\delta: 10.87(\mathrm{~s}, 1 \mathrm{H}), 8.12(\mathrm{~s}, 1 \mathrm{H}), 7.3(\mathrm{~d}, J=9.0 \mathrm{~Hz}, 1 \mathrm{H}), 6.93(\mathrm{~d}, J=9.0 \mathrm{~Hz}, 1 \mathrm{H}), 6.11(\mathrm{~s}, 1 \mathrm{H}), 2.51$ $\left(\mathrm{tt}, J_{1}=11.5 \mathrm{~Hz}, J_{2}=3.5 \mathrm{~Hz}, 1 \mathrm{H}\right), 2.40(\mathrm{~s} 3 \mathrm{H}), 2.01(\mathrm{~d}, J=13.5 \mathrm{~Hz}, 2 \mathrm{H}), 1.86\left(\mathrm{dt}, J_{1}=13.5 \mathrm{~Hz}, J_{2}=3.0 \mathrm{~Hz}\right.$, $2 \mathrm{H}), 1.58\left(\mathrm{qd}, J_{1}=12.0 \mathrm{~Hz}, J_{2}=3.0 \mathrm{~Hz}, 2 \mathrm{H}\right), 1.38-1.24(\mathrm{~m}, 4 \mathrm{H}) ;{ }^{13} \mathrm{C}$ NMR $\delta: 177.70,159.90,153.73$, 152.46, 145.67, 122.13, 116.37, 114.06, 112.54, 111.00, 45.83, 29.74, 25.44, 18.97; HR-ESI-MS m/z: 302.1384 $[\mathrm{M}+\mathrm{H}]^{+}$(calculated for $\mathrm{C}_{17} \mathrm{H}_{20} \mathrm{NO}_{4}, 302.1392$ ).

8-(2-Methyl-acryloylamino)-4-methylumbelliferone (4bg). White solid; yield: 20.8\%; m.p. 289.1-291.2 ${ }^{\circ} \mathrm{C}$; ${ }^{1} \mathrm{H}$ NMR $\delta: 10.75(\mathrm{~s}, 1 \mathrm{H}), 8.44(\mathrm{~s}, 1 \mathrm{H}), 7.35(\mathrm{~d}, J=9.0 \mathrm{~Hz}, 1 \mathrm{H}), 6.96(\mathrm{~d}, J=8.5 \mathrm{~Hz}, 1 \mathrm{H}), 6.12(\mathrm{~d}, J=7.0 \mathrm{~Hz}$, 2H), $5.66(\mathrm{~d}, J=1.0 \mathrm{~Hz}, 1 \mathrm{H}), 2.41(\mathrm{~s}, 3 \mathrm{H}), 2.17(\mathrm{~s}, 3 \mathrm{H}) ;{ }^{13} \mathrm{C}$ NMR $\delta: 168.62,159.57,153.57,152.58,145.90$, 138.06, 124.06, 122.41, 116.38, 113.78, 112.62, 111.12, 18.93, 18.59; HR-ESI-MS m/z: $260.0927[\mathrm{M}+\mathrm{H}]^{+}$ (calculated for $\mathrm{C}_{14} \mathrm{H}_{14} \mathrm{NO}_{4}, 260.0923$ ).

8-(Phenylacetylamino)-4-methylumbelliferone (4bh). White solid; yield: $39.2 \%$; m.p. $219.2-220.9{ }^{\circ} \mathrm{C} ;{ }^{1} \mathrm{H}$

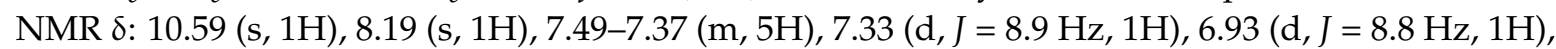
$6.09(\mathrm{~s}, 1 \mathrm{H}), 3.91(\mathrm{~s}, 2 \mathrm{H}), 2.38(\mathrm{~s}, 3 \mathrm{H}) ;{ }^{13} \mathrm{C}$ NMR $\delta: 172.56,159.44,153.35,152.29,145.75,133.11,129.52$, $129.48,128.21,122.34,116.24,113.80,112.57,111.16,43.74,18.89$;HR-ESI-MS $m / z: 310.1084\left[\mathrm{M}+\mathrm{H}^{+}\right.$ (calculated for $\mathrm{C}_{18} \mathrm{H}_{16} \mathrm{NO}_{4}, 310.1079$ ).

8-(Hydrocinnamoylamino)-4-methylumbelliferone (4bi). White solid; yield: $15.7 \%$; m.p. $237.5-239.0{ }^{\circ} \mathrm{C}$; ${ }^{1} \mathrm{H}$ NMR $\delta: 10.16(\mathrm{~s}, 1 \mathrm{H}), 8.17(\mathrm{~s}, 1 \mathrm{H}), 7.35(\mathrm{~d}, J=8.8 \mathrm{~Hz}, 1 \mathrm{H}), 7.32-7.27(\mathrm{~m}, 2 \mathrm{H}), 7.25-7.17(\mathrm{~m}, 3 \mathrm{H}), 6.95$ $(\mathrm{d}, J=8.8 \mathrm{~Hz}, 1 \mathrm{H}), 6.11(\mathrm{~s}, 1 \mathrm{H}), 3.10(\mathrm{t}, J=7.4 \mathrm{~Hz}, 2 \mathrm{H}), 2.91(\mathrm{t}, J=7.4 \mathrm{~Hz}, 2 \mathrm{H}), 2.40(\mathrm{~s}, 3 \mathrm{H}) ;{ }^{13} \mathrm{C}$ NMR $\delta$ : 172.94, 159.32, 152.98, 151.62, 144.63, 138.71, 127.68, 127.50, 127.30, 127.22, 125.54, 121.25, 115.48, 109.88, 37.36, 30.52, 17.91; HR-ESI-MS $m / z: 322.1077$ [M - H] ${ }^{-}$(calculated for $\mathrm{C}_{19} \mathrm{H}_{16} \mathrm{NO}_{4}, 322.1079$ ).

8-(Cinnamoylamino)-4-methylumbelliferone (4bj). Yellow solid; yield: $41.2 \%$; m.p. ${ }^{152.3-155.3 ~}{ }^{\circ} \mathrm{C}$;

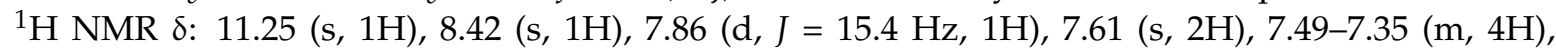
7.00-6.99 (m, 1H), $6.87(\mathrm{~d}, J=15.3 \mathrm{~Hz}, 1 \mathrm{H}), 6.16(\mathrm{~s}, 1 \mathrm{H}), 2.44(\mathrm{~s}, 3 \mathrm{H}) ;{ }^{13} \mathrm{C}$ NMR $\delta: 166.43,160.20,153.98$, 152.80, 145.70, 145.43, 134.02, 130.82, 129.09, 128.46, 122.38, 118.27, 116.57, 114.25, 112.57, 110.94, 19.01; HR-ESI-MS $m / z: 344.0901[\mathrm{M}+\mathrm{Na}]^{+}$(calculated for $\mathrm{C}_{19} \mathrm{H}_{15} \mathrm{NNaO}_{4}, 344.0899$ ).

8-(4-Methyl-benzoylamino)-4-methylumbelliferone (4bk). Yellow solid; yield: 16.8\%; m.p. 183.8-185.6 ${ }^{\circ} \mathrm{C} ;{ }^{1} \mathrm{H}$ NMR $\delta: 10.93(\mathrm{~s}, 1 \mathrm{H}), 8.78(\mathrm{~s}, 1 \mathrm{H}), 7.89(\mathrm{~d}, J=8.2 \mathrm{~Hz}, 2 \mathrm{H}), 7.37(\mathrm{~d}, J=8.9 \mathrm{~Hz}, 1 \mathrm{H}), 7.32(\mathrm{~d}$, $J=8.2 \mathrm{~Hz}, 2 \mathrm{H}), 6.98(\mathrm{~d}, J=8.8 \mathrm{~Hz}, 1 \mathrm{H}), 6.12(\mathrm{~s}, 1 \mathrm{H}), 2.44(\mathrm{~s}, 3 \mathrm{H}), 2.41(\mathrm{~s}, 3 \mathrm{H}) ;{ }^{13} \mathrm{C}$ NMR $\delta: 167.85,159.72$, 153.64, 152.68, 146.07, 144.08, 129.84, 129.35, 127.78, 122.35, 116.44, 114.12, 112.69, 111.13, 21.67, 18.97; HR-ESI-MS $m / z: 332.0899[\mathrm{M}+\mathrm{Na}]^{+}$(calculated for $\mathrm{C}_{18} \mathrm{H}_{15} \mathrm{NNaO}_{4}, 332.0899$ ).

8-(4-Methoxyl-benzoylamino)-4-methylumbelliferone (4bl). White solid; yield: $43.4 \%$; m.p. $151.2-154.2{ }^{\circ} \mathrm{C}$; ${ }^{1} \mathrm{H}$ NMR $\delta: 11.08(\mathrm{~s}, 1 \mathrm{H}), 8.69(\mathrm{~s}, 1 \mathrm{H}), 7.98(\mathrm{~d}, J=8.9 \mathrm{~Hz}, 2 \mathrm{H}), 7.39(\mathrm{~d}, J=8.9 \mathrm{~Hz}, 1 \mathrm{H}), 7.03(\mathrm{~d}, J=9.0,2 \mathrm{H})$, $7.01(\mathrm{~d}, J=8.5 \mathrm{~Hz}, 1 \mathrm{H}), 6.15(\mathrm{~s}, 1 \mathrm{H}), 3.90(\mathrm{~s}, 3 \mathrm{H}), 2.44(\mathrm{~s}, 3 \mathrm{H}) ;{ }^{13} \mathrm{C}$ NMR $\delta: 167.34,163.61,159.74,153.70$, 
152.57, 145.95, 129.81, 124.26, 122.21, 116.46, 114.45, 114.21, 112.66, 111.10, 55.63, 29.72; HR-ESI-MS m/z: $326.1036[\mathrm{M}+\mathrm{H}]^{+}$(calculated for $\left.\mathrm{C}_{18} \mathrm{H}_{16} \mathrm{NO}_{5}, 326.1028\right)$.

8-(3-Fluoro-benzoylamino)-4-methylumbelliferone (4bm). White solid; yield: $26.8 \%$; m.p. $261.7-263.2{ }^{\circ} \mathrm{C}$; ${ }^{1} \mathrm{H}$ NMR $\delta: 10.52$ (s, 1H), $8.79(\mathrm{~s}, 1 \mathrm{H}), 7.77\left(\mathrm{dq}, J_{1}=7.8 \mathrm{~Hz}, J_{2}=0.5 \mathrm{~Hz}, 1 \mathrm{H}\right), 7.72\left(\mathrm{dt}, J_{1}=8.8 \mathrm{~Hz}\right.$, $\left.J_{2}=2.5 \mathrm{~Hz}, 1 \mathrm{H}\right), 7.56-7.52(\mathrm{~m}, 1 \mathrm{H}), 7.42(\mathrm{~d}, J=8.9 \mathrm{~Hz}, 1 \mathrm{H}), 7.34\left(\mathrm{td}, J_{1}=8.6, J_{2}=3.3 \mathrm{~Hz}, 1 \mathrm{H}\right), 7.02(\mathrm{~d}$, $J=8.9 \mathrm{~Hz}, 1 \mathrm{H}), 6.16(\mathrm{~s}, 1 \mathrm{H}), 2.44(\mathrm{~s}, 3 \mathrm{H}) ;{ }^{13} \mathrm{C}$ NMR $\delta: 166.61,162.94(J=249.48 \mathrm{~Hz}), 159.61,153.66,152.65$, $146.15,134.47(J=7.56 \mathrm{~Hz}), 130.97(\mathrm{~J}=8.82 \mathrm{~Hz}), 123.00(J=2.52 \mathrm{~Hz}), 122.77,120.31(\mathrm{~J}=21.42 \mathrm{~Hz}), 116.57$, $115.42(J=23.94 \mathrm{~Hz}), 113.78,112.84,111.28,18.99$; HR-ESI-MS $m / z: 312.0676[\mathrm{M}-\mathrm{H}]^{-}$(calculated for $^{-}$ $\left.\mathrm{C}_{17} \mathrm{H}_{11} \mathrm{FNO}_{4}, 312.0672\right)$.

8-(3-Bromo-benzoylamino)-4-methylumbelliferone (4bn). White solid; yield: 40.7\%; m.p. $237.2-239.3{ }^{\circ} \mathrm{C}$; ${ }^{1} \mathrm{H}$ NMR $\delta: 10.44(\mathrm{~s}, 1 \mathrm{H}), 8.78(\mathrm{~s}, 1 \mathrm{H}), 8.17(\mathrm{~s}, 1 \mathrm{H}), 7.90(\mathrm{~d}, J=7.5 \mathrm{~Hz}, 1 \mathrm{H}), 7.76(\mathrm{~d}, J=7.8 \mathrm{~Hz}, 1 \mathrm{H})$, 7.49-7.37 (m, 2H), $7.03(\mathrm{~d}, J=8.8 \mathrm{~Hz}, 1 \mathrm{H}), 6.16(\mathrm{~s}, 1 \mathrm{H}), 2.44(\mathrm{~s}, 3 \mathrm{H}) ;{ }^{13} \mathrm{C}$ NMR $\delta: 166.51,159.62,153.66$, $152.65,146.16,136.14,134.20,131.27,130.63,125.83,123.45,122.81,116.60,113.78,112.86,111.30,19.00$; HR-ESI-MS m/z: 374.0025, 376.0016 [M + H] ${ }^{+}$(calculated for $\mathrm{C}_{17} \mathrm{H}_{13} \mathrm{BrNO}_{4}, 374.0028,376.0007$ ).

8-(Furyl-2-formylamino)-4-methylumbelliferone (4bo). White solid; yield: $23.4 \%$; m.p. $280.1-281.5{ }^{\circ} \mathrm{C}$; ${ }^{1} \mathrm{H}$ NMR $\delta: 10.77$ (s, 1H), 8.96 (s, 1H), $7.67(\mathrm{~s}, 1 \mathrm{H}), 7.40(\mathrm{~d}, J=9.0 \mathrm{~Hz}, 1 \mathrm{H}), 7.37$ (d, J = 3.5 Hz, $\left.1 \mathrm{H}\right), 7.00$ (d, $J=9.0 \mathrm{~Hz}, 1 \mathrm{H}), 6.63(\mathrm{~s}, 1 \mathrm{H}), 6.16(\mathrm{~s}, 1 \mathrm{H}), 2.43(\mathrm{~s}, 3 \mathrm{H}) ;{ }^{13} \mathrm{C} \mathrm{NMR} \delta: 159.61,157.82,153.45,152.34,146.17$, 145.99, 145.94, 122.43, 117.76, 116.40, 113.57, 113.05, 112.77, 111.30, 18.92;HR-ESI-MS m/z: 284.0564 $[\mathrm{M}-\mathrm{H}]^{-}$(calculated for $\left.\mathrm{C}_{15} \mathrm{H}_{10} \mathrm{NO}_{5}, 284.0559\right)$.

8-(Thienyl-2-formylamino)-4-methylumbelliferone (4bp). White solid; yield: 29.2\%; m.p. 244.6-246.2 ${ }^{\circ} \mathrm{C}$;

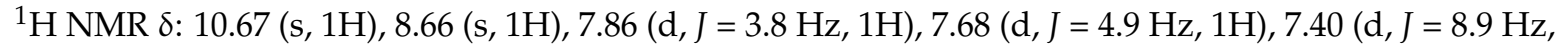
$1 \mathrm{H}), 7.20(\mathrm{t}, J=4.5 \mathrm{~Hz}, 1 \mathrm{H}), 7.01(\mathrm{~d}, J=8.9 \mathrm{~Hz}, 1 \mathrm{H}), 6.15(\mathrm{~s}, 1 \mathrm{H}), 2.43(\mathrm{~s}, 3 \mathrm{H}) ;{ }^{13} \mathrm{C}$ NMR $\delta: 162.21$, 159.60, 153.63, 152.36, 145.84, 136.42, 133.09, 130.56, 128.44, 122.42, 116.47, 113.81, 112.75, 111.20, 18.97; HR-ESI-MS m/z: $302.0491[\mathrm{M}+\mathrm{H}]^{+}$(calculated for $\mathrm{C}_{15} \mathrm{H}_{12} \mathrm{NO}_{4} \mathrm{~S}, 302.0487$ ).

8-(Naphthyl-1-formylamino)-4-methylumbelliferone (4bq). White solid; yield: 41.2\%; m.p. 208.5-210.9 ${ }^{\circ} \mathrm{C}$; ${ }^{1} \mathrm{H}$ NMR $\delta: 10.69(\mathrm{~s}, 1 \mathrm{H}), 8.63(\mathrm{~s}, 1 \mathrm{H}), 8.44(\mathrm{~d}, J=8.4 \mathrm{~Hz}, 1 \mathrm{H}), 8.07(\mathrm{~d}, J=8.3 \mathrm{~Hz}, 1 \mathrm{H}), 7.94(\mathrm{t}, J=7.3 \mathrm{~Hz}$, $2 \mathrm{H}), 7.66(\mathrm{t}, J=7.0 \mathrm{~Hz}, 1 \mathrm{H}), 7.59(\mathrm{q}, J=7.5 \mathrm{~Hz}, 2 \mathrm{H}), 7.45(\mathrm{~d}, J=8.9 \mathrm{~Hz}, 1 \mathrm{H}), 7.08(\mathrm{~d}, J=8.9 \mathrm{~Hz}, 1 \mathrm{H}), 6.15$ (s, 1H), 2.45 (s, 3H); ${ }^{13} \mathrm{C}$ NMR $\delta: 170.31,159.70,153.60,152.68,146.12,133.87,132.64,131.46,130.06$, $128.72,128.02,126.97,126.61,124.92,124.80,122.71,116.61,114.32,112.89,111.30,19.00$; HR-ESI-MS $m / z: 346.1079[\mathrm{M}+\mathrm{H}]^{+}$(calculated for $\mathrm{C}_{21} \mathrm{H}_{16} \mathrm{NO}_{4}, 346.1079$ ).

\subsection{Procedures for ActivityEvaluation}

\subsubsection{Acaricidal Activity}

The acaricidal activities of the title compounds against the phytophagous mite T. cinnabarinus were evaluated by the microimmersion bioassay method [45]. Each title compound was dissolved by acetone (for $\mathbf{4 b a}-\mathbf{4 b q}$ ) or DMSO/ $\mathrm{H}_{2} \mathrm{O}(1: 1$, for $\mathbf{4 a a}-\mathbf{4 a h})$ to give a $10 \mathrm{~g} / \mathrm{L}$ stock solution. Then 0.1 and $0.5 \mathrm{~mL}$ of the stock solution were diluted separately to $5 \mathrm{~mL}$ by an appropriate amount of acetone $\left(\mathrm{DMSO} / \mathrm{H}_{2} \mathrm{O}\right)$ and $0.1 \%$ Tween 80 in order to achieve the $0.2 \mathrm{~g} / \mathrm{L}$ and $1 \mathrm{~g} / \mathrm{L}$, respectively, of test compound in acetone (DMSO/ $\mathrm{H}_{2} \mathrm{O}$ )-0.1\% Tween 80 (3:7). Ten test mites were sucked into the terminal pipette tip by a sucking trap, then $150 \mu \mathrm{L}$ of the compound solution was sucked in to immerse the mites for 15 seconds. After the exposure, the mites were carefully removed onto a piece of filter paper in order to air dry the body surface. At the same time, the petiole of a fresh peanut leaf was wrapped around a ball of absorbent cotton, which was then wetted and sealed with a piece of silver paper. The hydrated leaf was dipped into the same compound solution for $5 \mathrm{~s}$ and taken out to air dry, as with the mites. Then the treated mites and peanut leaf were sealed in one well of a six pore plate with a piece of rice paper. Finally the mites were reared in an illumination incubator set at $28 \pm 1{ }^{\circ} \mathrm{C}$ and $50-55 \%$ relative humidity in alternating $12 \mathrm{~h}$ of light and $12 \mathrm{~h}$ of dark for $72 \mathrm{~h}$. Acetone (DMSO/ $\left.\mathrm{H}_{2} \mathrm{O}\right)-0.1 \%$ Tween 80 
(3:7) was used as a blank control, and Bifenazate was used as a positive control. Each treatment was conducted in four replicates. The dead mites were recorded every $24 \mathrm{~h}$ under a stereoscope and the corrected mortalities of $T$. cinnabarinus treated by the compounds were calculated.

\subsubsection{Herbicidal Activity}

The herbicidal activities of the synthesized compounds against the taproot and caulis development of the dicotyledonous weed C. album and the monocotyledonous weed D. sanguinalis were determined in vitro [38]. A suspension of $5 \mathrm{~g}$ agar powder in $1 \mathrm{~L}$ distilled water was heated to melt, and then cooled to $40-50^{\circ} \mathrm{C}$. Each title compound was dissolved by acetone to give a stock solution of $10 \mathrm{~g} / \mathrm{L}$, and 0.1 or $0.5 \mathrm{~mL}$ of the stock solution was added to $50 \mathrm{~mL}$ of the melting agar at $45^{\circ} \mathrm{C}$ to achieve the required concentrations. Then, $5 \mathrm{~mL}$ of the agar-containing compound was added to a beaker $(10 \mathrm{~mL})$ and cooled, and uniform germinating seeds were placed on the surface of the agar mass. The beaker was sealed by a piece of plastic wrap with several small holes on it, and then the cultivations were carried out in an illumination incubator set at $28 \pm 1{ }^{\circ} \mathrm{C}$ and $50-55 \%$ relative humidity in alternating $12 \mathrm{~h}$ of light and $12 \mathrm{~h}$ of dark for 3 days. Acetone was used as a blank control, and Acetochlor was used as a positive control. Each treatment was conducted in three replicates. After 3 days of cultivation, the taproot and caulis lengths were measured, and the growth inhibitory rate related to the untreated control was determined.

\subsubsection{Antifungal Activity}

The antifungal activities of the title compounds against B. cinerea, $C$. gloeosporioides, A. brassicae and G. gramini were tested in vitro using the mycelium growth rate test [46]. The test compound was dissolved in acetone to form a series of proper concentration solutions. Then $1 \mathrm{~mL}$ of the solution was added to $100 \mathrm{~mL}$ melting potato dextrose agar (PDA) at $45^{\circ} \mathrm{C}$, and the mixture was shaken up to obtain the required concentration of the poisoned medium. $5 \mathrm{~mL}$ of the poisoned medium was poured into a $6 \mathrm{~cm}$ petri dish and cooled to room temperature to get a solid plate. After that, a $4 \mathrm{~mm}$ activated mycelium disk was inoculated on the PDA plate and incubated in the dark at $28{ }^{\circ} \mathrm{C}$ for $48 \mathrm{~h}$. The mycelium elongation radius $(\mathrm{mm})$ of the fungus settlements was measured and the growth inhibition rate related to the untreated control was calculated. Acetone was used as a blank control, and Carbendazim was used as a positive control. Each treatment was repeated for 3 times.

\section{Conclusions}

Twenty-five 4-methylumbelliferone amide derivatives were synthesized and their acaricidal, herbicidal and antifungal activities were evaluated. Compounds 4bi [8-(hydrocinnamoylamino)], 4ac [6-(2-methyl-acryloylamino)] and $4 \mathbf{b d}$ [8-(4-chloro-butanoylamino)] were strongly acaricidal against T. cinnabarinus, with $72 \mathrm{~h}$ corrected mortalities of greater than $80 \%$ at $1000 \mathrm{mg} / \mathrm{L}$. Compounds $4 \mathrm{bh}$ [8-(phenylacetylamino)] and $4 \mathbf{b f}$ [8-(cyclohexyl-formylamino)] exhibited the strongest inhibition against the taproot development of $D$. sanguinalis and $C$. glaucum, and were more potent than the commercial herbicide Acetochlor to D. sanguinalis. Moreover, compounds $4 \mathbf{b k}$ [8-(4-methylbenzoylamino)], $\mathbf{4 b h}$ [8-(phenylacetylamino)] and $\mathbf{4 b p}$ [8-(thienyl-2-formylamino)] showed the highest antifungal activities against the mycelium growth of $V$. mali, and were more effective than the commercial fungicide Carbendazim.

Supplementary Materials: The following are available online.

Acknowledgments: This work was supported by the National Natural Science Foundation of China (No. 31471808) and partially by Chinese National Undergraduate Training Program for Innovation (No. 200610435032) under Grant.

Author Contributions: S.-H.H. conceived and designed the experiments; K.-L.M. and Y.W. performed the experiments and analyzed the data; S.-H.H. and Y.W. wrote the paper; Y.W. provided reagents/materials.

Conflicts of Interest: The authors declare no conflict of interest. 


\section{References}

1. Ahmed, A. Coumarin Synthesis, Reactions and Pharmacological Activities; LAP LAMBERT Academic Publishing: Saarbrücken, Germany, 2013; ISBN 3659503603.

2. Zhang, G.H.; Zheng, H.; Guo, M.Y.; Du, L.; Liu, G.J.; Wang, P. Synthesis of polymeric fluorescent brightener based on coumarin and its performances on paper as light stabilizer, fluorescent brightener and surface sizing agent. Appl. Surf. Sci. 2016, 367, 167-173. [CrossRef]

3. Mishra, V.R.; Sekar, N. Photostability of coumarin laser dyes-A mechanistic study using global and local reactivity descriptors. J. Fluoresc. 2017, 27, 1101-1108. [CrossRef] [PubMed]

4. Lei, L.; Xue, Y.B.; Liu, Z.; Peng, S.S.; He, Y.; Zhang, Y.; Fang, R.; Wang, J.P.; Luo, Z.W.; Yao, G.M.; et al. Coumarin derivatives from Ainsliaea fragrans and their anticoagulant activity. Sci. Rep. 2015, 5, 13544. [CrossRef] [PubMed]

5. Bertin, R.; Chen, Z.; Martínez-Vázquez, M.; García-Argaéz, A.; Froldi, G. Vasodilation and radical-scavenging activity of imperatorin and selected coumarinic and flavonoid compounds from genus Casimiroa. Phytomedicine 2014, 21, 586-594. [CrossRef] [PubMed]

6. Kuk, H.S.; Won, S.W. Effects of psoralen and angelicin on hepatic drug-metabolizing enzyme activities. Arch. Pharm. Res. 1988, 11, 122-126.

7. Srivastava, P.; Vyas, V.K.; Variya, B.; Patel, P.; Qureshi, G.; Ghate, M. Synthesis, anti-inflammatory, analgesic, 5-lipoxygenase (5-LOX) inhibition activities, and molecular docking study of 7-substituted coumarin derivatives. Bioorg. Chem. 2016, 67, 130-138. [CrossRef] [PubMed]

8. Jacquot, Y.; Laïos, I.; Cleeren, A.; Nonclercq, D.; Bermont, L.; Refouvelet, B.; Boubekeur, K.; Xicluna, A.; Leclercq, G.; Laurent, G. Synthesis, structure, and estrogenic activity of 4-amino-3-(2-methylbenzyl) coumarins on human breast carcinoma cells. Bioorg. Med. Chem. 2007, 15, 2269-2282. [CrossRef] [PubMed]

9. Renuka, N.; Pavithra, G.; Ajay, K.K. Synthesis of coumarin appended 1,3-oxazines as potent antimicrobial and antioxidant agents. Pharm. Chem. J. 2017, 51, 582-589.

10. Kumar, R.; Banerjee, S.K.; Handa, K.L. Coumarins of Heracleum canescens and Heracleum pinnatum. Sources for dermal photosensitizing agents. PlantaMedica 1976, 30, 291-295.

11. Shakeel-u-Rehman; Khan, R.; Bhat, K.; Raja, A.; Shawl, A.; Alam, M. Isolation, characterisation and antibacterial activity studies of coumarins from Rhododendron lepidotum Wall. ex G. Don, Ericaceae. Braz. J. Pharmacogn. 2010, 20, 886-890. [CrossRef]

12. Song, P.P.; Zhao, J.; Liu, Z.L.; Duan, Y.B.; Hou, Y.P.; Zhao, C.Q.; Wu, M.; Wei, M.; Wang, N.H.; Lv, Y.; et al. Evaluation of antifungal activities and structure-activity relationships of coumarin derivatives. Pest Manag. Sci. 2017, 73, 94-101. [CrossRef] [PubMed]

13. Liu, G.L.; Hu, Y.; Chen, X.H.; Wang, G.X.; Ling, F. Synthesis and anthelmintic activity of coumarin-imidazole hybrid derivatives against Dactylogyrus intermedius in goldfish. Bioorg. Med. Chem. Lett. 2016, 26, 5039-5043. [CrossRef] [PubMed]

14. Wu, C.J.; Wang, J.B.; Shen, J.J.; Bi, C.; Zhou, H.W. Coumarin-based $\mathrm{Hg}^{2+}$ fluorescent probe: Synthesis and turn-on fluorescence detection in neat aqueous solution. Sens. Actuators B Chem. 2017, 243, 678-683. [CrossRef]

15. Pang, L.; Zhou, Y.; Gao, W.; Song, H.; Wang, X.; Wang, Y. A highly selective and sensitive fluorescence probe for rapid detection of hypochlorite in tap water and cancer cells. RSC Adv. 2016, 6, 105795-105800. [CrossRef]

16. Anna, Z.; Dominik, K.; Filip, B.; Ryszard, O. Mixed carbonates as useful substrates for a fluorogenic assay for lipases and esterases. ChemBioChem 2015, 16, 677-682.

17. Arora, P.; Ranawat, M.S.; Arora, N. Synthesis and screening of some novel coumarin derivatives for antipsychotic activity. Res. J. Pharm. Technol. 2012, 5, 968-972.

18. Ostrowska, K.; Młodzikowska, K.; Głuch-Lutwin, M.; Gryboś, A.; Siwek, A. Synthesis of a new series of aryl/heteroarylpiperazinyl derivatives of 8-acetyl-7-hydroxy-4-methylcoumarin with low nanomolar 5-HT 1A affinities. Eur. J. Med. Chem. 2017, 137, 108-116. [CrossRef] [PubMed]

19. Nam, S.T.; Park, Y.H.; Kim, H.W.; Kim, H.S.; Lee, D.; Lee, M.B.; Kim, Y.M.; Choi, W.S. Suppression of IgE-mediated mast cell activation and mouse anaphylaxis via inhibition of Syk activation by 8-formyl-7-

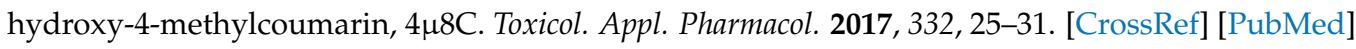


20. Hu, X.J.; Wang, X.B.; Kong, L.Y. $\alpha$-Glucosidase inhibitors via green pathway: Biotransformation for bicoumarins catalyzed by Momordica charantia peroxidase. J. Agric. Food Chem. 2013, 61, 1501-1508. [CrossRef] [PubMed]

21. Zhang, Y.; Zou, B.; Chen, Z.; Pan, Y.; Wang, H.; Liang, H.; Yi, X. Synthesis and antioxidant activities of novel 4-Schiff base-7-benzyloxy-coumarin derivatives. Bioorg. Med. Chem. Lett. 2011, 21, 6811-6815. [CrossRef] [PubMed]

22. Radwan, E.; Reem, I.A. Anti-inflammatory screening and molecular modeling of some novel coumarin derivatives. Molecules 2015, 20, 5374-5391.

23. Li, Y.J.; Wang, C.Y.; Ye, M.Y.; Yao, G.Y.; Wang, H.S. Novel coumarin-containing aminophosphonatesas antitumor agent: Synthesis, cytotoxicity, DNA-binding and apoptosis evaluation. Molecules 2015, 20, 14791-14809. [CrossRef] [PubMed]

24. Li, Y.J.; Ye, M.Y.; Huang, R.Z.; Yao, G.Y.; Pan, Y.M.; Liao, Z.X.; Wang, H.S. Coumarin-containing aminophosphonates bridged with chiral side chain: Synthesis and influence of chirality on cytotoxicity and DNA binding. Med. Chem. Res. 2014, 23, 3144-3156. [CrossRef]

25. Cong, N.T.; Nhan, H.T.; Van, H.L.; Thang, T.D.; Kuo, P.C. Synthesis and antibacterial activity of analogs of 5-arylidene-3-(4-methylcoumarin-7-yloxyacetylamino)-2-thioxo-1,3-thiazoli-din-4-one. Molecules 2014, 19, 13577-13586. [CrossRef] [PubMed]

26. Šarkanj, B.; Molnar, M.; Cacic, M.; Gille, L. 4-Methyl-7-hydroxycoumarin antifungal and antioxidant activity enhancement by substitution with thiosemicarbazide and thiazolidinone moieties. Food Chem. 2013, 139, 488-495. [CrossRef] [PubMed]

27. Benitez, A.; Yates, T.; Shamaldevi, N.; Bowen, T.; Lokeshwa, V. Dietary supplement hymecromone and sorafenib: A novel combination for the control of renal cell carcinoma. J. Urol. 2013, 190, 285-290. [CrossRef] [PubMed]

28. Nagy, N.; Kuipers, H.; Frymoyer, A.; Ishak, H.; Bollyky, J.; Wight, T.; Bollyky, P. 4-Methylumbelliferone treatment and hyaluronan inhibition as a therapeutic strategy in inflammation, autoimmunity, and cancer. Front. Immunol. 2015, 123, 1-11. [CrossRef] [PubMed]

29. Pan, L.; Li, X.Z.; Yan, Z.Q.; Guo, H.R.; Qin, B. Phytotoxicity of umbelliferone and its analogs: Structure-activity relationships and action mechanisms. Plant Physiol. Biochem. 2015, 97, 272-277. [CrossRef] [PubMed]

30. Pan, L.; Li, X.; Jin, H.; Yang, X.; Qin, B. Antifungal activity of umbelliferone derivatives: Synthesis and structure-activity relationships. Microb. Pathog. 2017, 104, 110-115. [CrossRef] [PubMed]

31. Deshmukh, M.; Pawar, P.; Joseph, M.; Phalgune, M.; Kashalkar, U.; Deshpande, R.; Nirmala, R. Efficacy of 4-methy-7-hydroxy coumarin derivatives against vectors Aedesa egypti and Culexquinque fasciatus. Indian J. Exp. Biol. 2008, 46, 788-792. [PubMed]

32. Huang, H.Z.; Yao, H.W.; Liu, J.Y.; Aman, I.S.; Shizuo, G.K.; Anthony, J.C.; Bruce, D.H. Development of pyrethroid-like fluorescent substrates for glutathione S-transferase. Anal. Biochem. 2012, 431, 77-83. [CrossRef] [PubMed]

33. Chetan, T.P.; Sangamesh, A.P.; Ajaykumar, D.K.; Vinod, H.N.; Manjunathad, M.; Shivshankar, M.K.; Prema, S.B. Synthesis, spectral, thermal, fluorescence, antimicrobial, anthelmintic and DNA cleavage studies of mononuclear metal chelates of bi-dentate $2 \mathrm{H}$-chromene-2-one Schiff base. J. Photochem. Photobiol. B 2015, 148, 322-332.

34. Tang, C.C.; Li, Y.C.; Chen, B.; Yang, H.Z.; Jin, G.Y. Pesticide Chemistry; Nankai University: Tianjin, China, 1998; ISBN 7-310-01010-8. (In Chinese)

35. Qiao, L.L.; Wei, Y.; Hao, S.H. Synthesis and biological activity of novel fluorinated amide hydroxy methyl coumarin derivatives. Chin. J. Org. Chem. 2017. (In Chinese) [CrossRef]

36. Wang, D.; Wei, Y.; Hao, S.H. Synthesis and Herbicidal Activity of N-Acyl-N-(m-fluoro-benzyl)-6-aminocoumarin. Chin. J. Organ. Chem. 2015, 35, 1691-1699. (In Chinese) [CrossRef]

37. Shah, N.M.; Mehta, D.H. Nitration of 7-hydroxy-4-methylcoumarin and its methyl ether. J. Indian Chem. Soc. 1954, 31, 784-786.

38. Banal, V.; Khanna, R.N. Regioselective mononitration of coumarins using chromium nitrate as nitrating agent. Synth. Commun. 2002, 32, 1345-1350. [CrossRef]

39. Ramana, M.M.V.; Malik, S.S.; Parihar, J.A. Guanidinium nitrate: A novel reagent for aryl nitrations. Tetrahedron Lett. 2004, 45, 8681-8683. [CrossRef] 
40. Ganguly, N.; Sukai, A.K.; De, S. Cerium (IV) ammonium nitrate mediated nitration of coumarins. Synth. Commun. 2001, 31, 301-309. [CrossRef]

41. Kozlova, I.K. Synthesis of o-acylamino-4-methyl-7-hydroxycoumarins (4-methylumbelliferones). Chem. Heterocycl. Compd. 1985, 21, 750-753. [CrossRef]

42. Tyagi, Y.K.; Kumar, A.; Raj, H.G.; Vohra, P.; Gupta, G.; Kumari, R.; Kumar, P.; Gupta, R.K. Synthesis of novel amino and acetyl amino-4-methylcoumarins and evaluation of their antioxidant activity. Eur. J. Med. Chem. 2005, 40, 413-420. [CrossRef] [PubMed]

43. Chaudhari, H.K.; Pahelkar, A.; Takale, B.S. Preparative-scale synthesis of amino coumarins through new sequential nitration and reduction protocol. Tetrahedron Lett. 2017, 58, 4107-4110. [CrossRef]

44. Hao, S.H.; Miao, K.L.; Wei, Y.; Wang, T. Synthesis and Acaricidal Activity of a Kind of Novel Amidating Hydroxymethyl Coumarin Derivatives. CN 107011306A; 4 August 2017. Available online: http:/ / epub.sipo. gov.cn/pam.action (accessed on 20 December 2017). (In Chinese)

45. Dennehy, T.J.; Farnham, A.W.; Denholm, I. The microimmersion bioassay: A novel method for the topical application of pesticides to spider mites. Pest Manag. Sci. 2010, 39, 47-54. [CrossRef]

46. Wei, Y.; Li, S.Q.; Hao, S.H. New angular oxazole-fused coumarin derivatives: Synthesis and biological activities. Nat. Prod. Res. 2017. [CrossRef] [PubMed]

Sample Availability: Samples of the compounds are not available from the authors. 